\title{
Geoacoustic Seafloor Exploration with a Towed Array in a Shallow Water Area of the Strait of Sicily
}

\author{
Andrea Caiti, Member, IEEE, Sérgio M. Jesus, and Åge Kristensen
}

\begin{abstract}
Acoustic propagation in shallow water is greatly dependent on the geoacoustic properties of the seabottom. This paper exploits this dependence for estimating geoacoustic sediment properties from the bottom acoustic returns of known signals received on a hydrophone line array. There are two major issues in this approach: one is the feasibility of acoustic inversion with a limited aperture line array, the other is related to the knowledge of the geometry of the experimental configuration. To test the feasibility of this approach, a 40-hydrophone-4-m spaced towed array together with a low-frequency acoustic source, was operated at a shallow water site in the Strait of Sicily. In order to estimate the array deformation in real time, it has been equipped with a set of nonacoustic positioning sensors (compasses, tiltmeters, pressure gauges). The acoustic data were inverted using two complementary approaches: a genetic algorithm (GA) like approach and a radial basis functions (RBF) inversion scheme. More traditional methods, based on core sampling, seismic survey and geophone data, together with Hamilton's regression curves, have also been employed on the same tracks, in order to provide a ground truth reference environment. The results of the experiment, can be summarized as follows: 1) the towed array movement is not negligible for the application considered and the use of positioning sensors are essential for a proper acoustic inversion, 2) the inversion with $\mathrm{GA}$ and $\mathrm{RBF}$ are in good qualitative agreement with the ground truth model, and 3) the GA scheme tends to have better stability properties. On the other hand, repeated inversion of successive field measurements requires much less computational effort with $\mathrm{RBF}$.
\end{abstract}

\section{INTRODUCTION}

$\mathbf{S}$ EAFLOOR sediments in shallow water areas are most often characterized by strong variability, both in terms of sediment properties and of layer thickness. Seismic surveying is currently used for determining layer thickness variability along selected tracks while the morphological changes of the different strata are estimated from a number of in situ samples (typically, cores) strategically placed along the tracks. Employing seismic surveying and coring for exploring extensive areas is, in general, a very expensive and timeconsuming task. A possible way to circumvent this problem is to extend the concept of the existing seismic survey systems and attempt to directly invert the acoustic field received on a towed array of hydrophones avoiding the need for coring. Inversion of acoustic returns for estimating seabottom geoacoustic parameters has been proposed by Frisk et al. [1] and then attempted with different approaches and in various

Manuscript received February 10, 1996; revised July 30, 1996.

A. Caiti is with Dist-Universita di Genova, IT-16145 Genoa, Italy.

S. M. Jesus is with the Universidade do Algave, Campus de Gambelas, 8000 Faro, Portugal.

$\AA$. Kristensen is wih Statoil, Trondheim, Norway.

Publisher Item Identifier S 0364-9059(96)07982-4 environments by others [2]-[4]. An overview of the work done to date in this field can be found in [5] and in the references herein.

The approach discussed here differs from most common approaches proposed to date both in the system geometry and in its mode of operation. In particular, the use of a horizontal line array and a sound source towed by the same ship imposes a constant source-receiver range and physical limitations in the usable array aperture for safe operation in shallow water. Another important difference is that the bottom reflected acoustic field measured at a given instant in time corresponds to a limited seafloor portion between the source and the receiving hydrophone array allowing, under a successful inversion, for a spatially localized geoacoustic estimate. This is in contrast with most of the methods described in the literature that provide average geoacoustic estimates over several kilometers.

There are two major issues in this approach: one is the feasibility of acoustic inversion with a limited aperture line array, the other is the need for a precise knowledge of the geometry of the experimental configuration and, in particular, for the relative position of each receiver in the array with respect to the sound source. To test the feasibility of this approach, a towed array, consisting of 40 hydrophones at $4 \mathrm{~m}$ spacing, together with a low-frequency acoustic source, was operated in a shallow water site in the Strait of Sicily along a selected track. This $156-\mathrm{m}$ aperture array was equipped with a set of nonacoustic positioning sensors (compasses, tiltmeters, pressure gauges) in order to estimate the array deformation in real time, and to estimate the source-receiver geometry. Using the estimated geometry and the available environmental information, the acoustic data gathered was inverted using two approaches; a genetic algorithm $(\mathrm{GA})$ and a radial basis function ( $\mathrm{RBF}$ ) inversion scheme. More traditional approaches, based on core sampling, seismic survey and geophone data, together with Hamiliton's regression curves, were also employed along the same track, in order to provide a ground truth reference environment model.

This paper is organized as follows. The next section presents a full description of the sea trial and the measurements performed at the experimental site for estimating the ground truth environment model. Section III describes the towed array nonacoustic instrumentation for array deformation estimation, the methods employed, and the relevant features extracted. Section IV deals with the data inversion methods and seafloor 


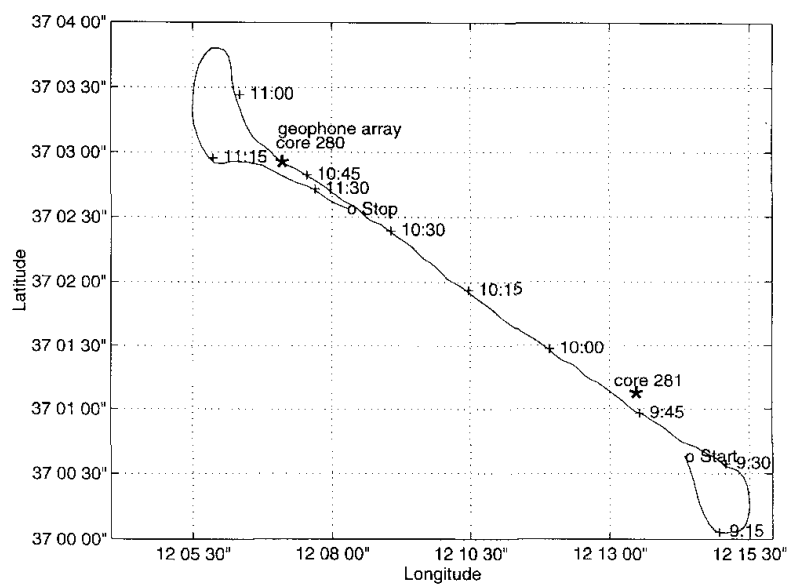

Fig. 1. Ship navigation track during SAG1-94 sea trial.

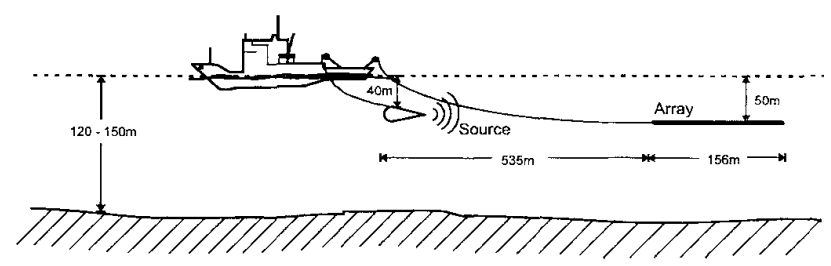

Fig. 2. Source-receiver experimental setup.

model identification. Section $\mathrm{V}$ shows and discusses the results obtained at the Adventure Bank site in the Strait of Sicily area and Section VI draws some conclusions and future trends.

\section{EXPERIMENTAL SETUP}

\section{A. Experimental Area and the Towed Array Run}

The SAG1-94 experiment took place at the Adventure Bank, Strait of Sicily, during March of 1994 (Fig. 1). The objective of this experiment was to test the possibility of estimating the bottom geoacoustic parameters by inversion of towed array acoustic data in a weakly geoacoustic range-dependent area. The source-receiver geometry used during the experiment is schematically depicted in Fig. 2. The sound speed in the water column was measured with CTD and XBT's casts, before and during the acoustic experiment, as well as on the other days during the same cruise. The result was a very stable typical winter Mediterranean sound velocity profile, slightly upward refracting with $1507 \mathrm{~m} / \mathrm{s}$ at the surface and $1510 \mathrm{~m} / \mathrm{s}$ near the bottom.

The towed array was equipped with both acoustic and nonacoustic sensors. The acoustic portion of the array consisted of 40 omnidirectional hydrophones with a spacing of 4 $\mathrm{m}$ including adjustable prefiltering and acquisition rate. The nonacoustic sensors are described in the next section.

The array was towed at a speed of 4 knots and at a depth of approximately $50 \mathrm{~m}$. The measured water depth along the track is represented in Fig. 3. This figure shows two distinct portions: one that has a relatively constant water depth of approximately $120 \mathrm{~m}$ and another that has a variable

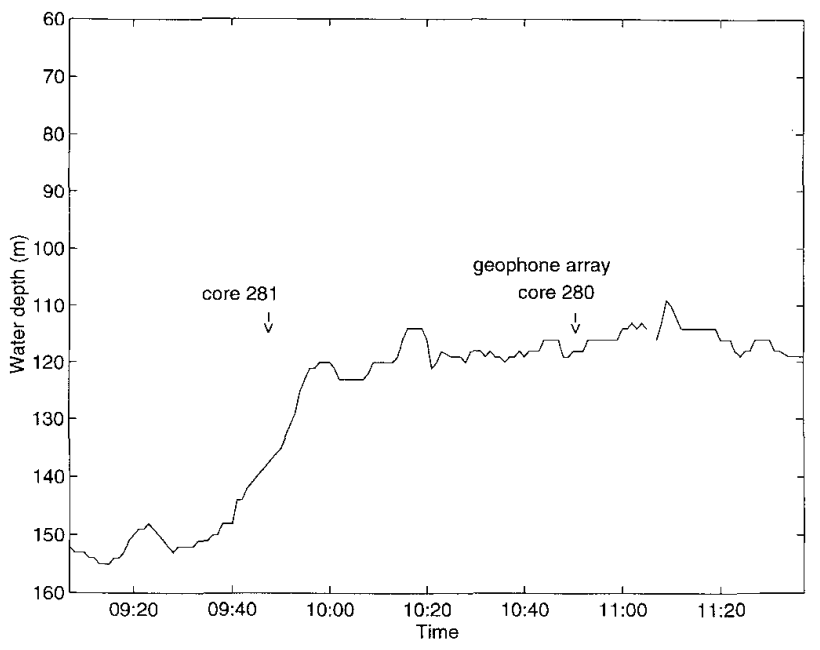

Fig. 3. Measured water depth along ship track with positions of cores and geophone array.

depth between 120-150-m depth. The acoustic source, a flextensional transducer with a lower resonance at $120 \mathrm{~Hz}$, was suspended at approximately $40-\mathrm{m}$ depth. A pressure gauge installed on the transducer allowed to precisely monitoring of the source depth along the track.

Two different kinds of acoustic signals were transmitted during the tow.

- Continuous wave (CW) signals at a given frequency, to be used in the inversion schemes. The track was divided into several runs each lasting $5 \mathrm{~min}$ (for convenience of analysis). Those that have been analyzed in this paper are listed in Table I, together with the transmitted frequency. When the source was transmitting in CW mode, each hydrophone output was low pass filtered at $300 \mathrm{~Hz}$, sampled at $750 \mathrm{~Hz}$, and coded with 12 bits. The received time series at each hydrophone was divided in portions of 2048 samples (roughly corresponding to $3 \mathrm{~s}$ of data) and transformed into the frequency domain, with a standard fast Fourier transform (FFT). From the transformed data the received acoustic field (amplitude and phase) at the transmitting frequency is extracted for each hydrophone. In this way it is possible to build, for every 3-s interval, the received acoustic field at the hydrophone positions. In the following we refer to these data as the "snapshot" of the acoustic field.

- Triangularly shaped acoustic pulses, of 2.5-ms length, to monitor the source-receiving distance. At certain intervals along the track (typically every $15 \mathrm{~min}$, but the sequence is not regular), the source was switched to the pulse mode, the low pass filter at the hydrophone output switched to $500 \mathrm{~Hz}$, and the sampling rate changed to $1000 \mathrm{~Hz}$. Each impulse transmitted is referred to as "ping" data in the following. The ping data acquisition lasted for about 5 min (including the time necessary to switch from one transmitting-receiving configuration to the other), and then $\mathrm{CW}$ transmission and acquisition was resumed. From the acoustic pings, the range between the source and the first array hydrophone was computed through a 


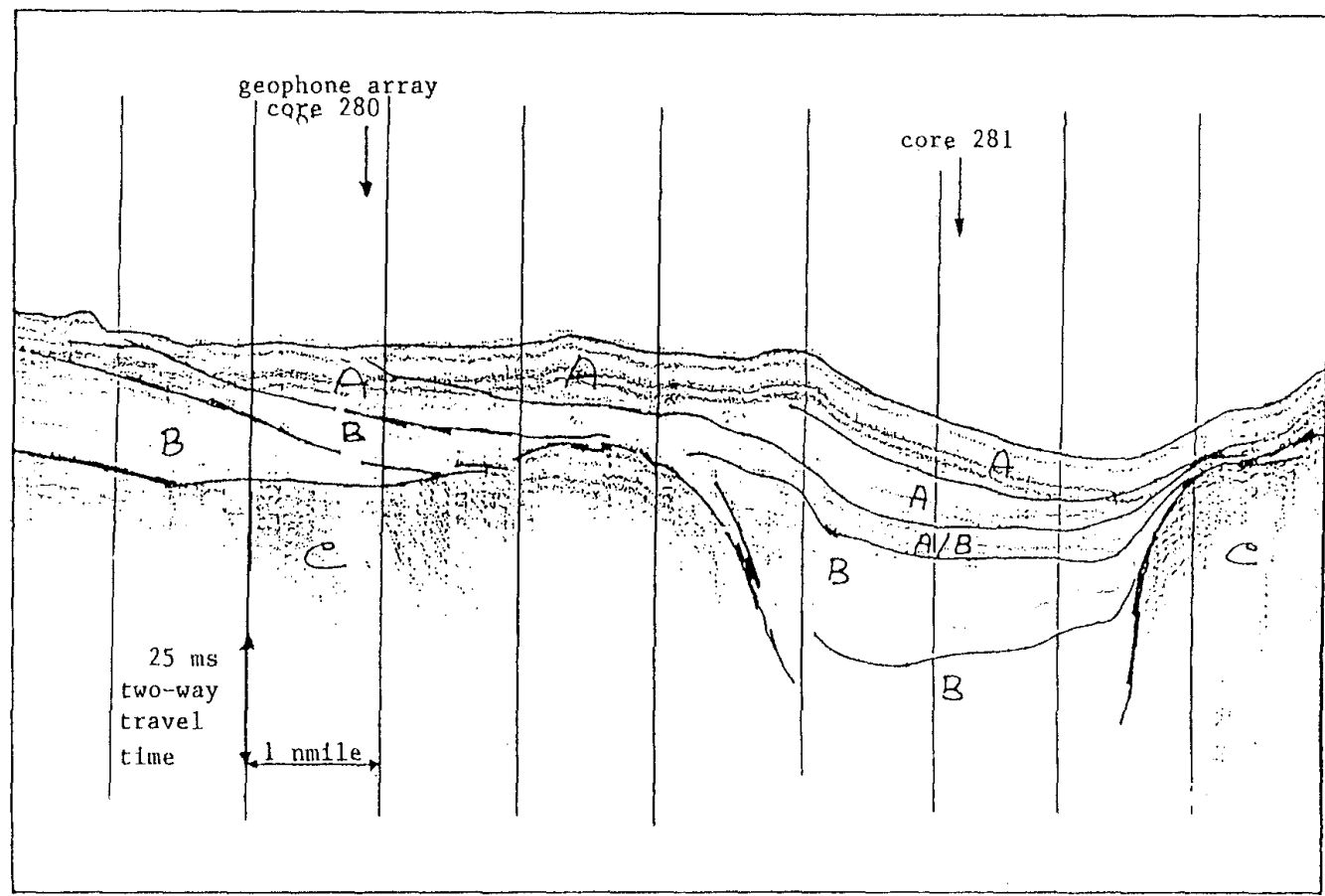

Fig. 4. Seismic survey along towed array track with geological interpretation. $(A)$ Recent sediments, $(B)$ Quartenary sediments. $(C)$ Miocene sedimentary rocks.

TABLE I

List of Runs During Day 4 March 1994, SAG1-94

\begin{tabular}{|c|c|c|c|c|c|}
\hline $\begin{array}{c}\text { Run } \\
\#\end{array}$ & $\begin{array}{c}\text { Start } \\
\text { hh:mm }\end{array}$ & $\begin{array}{c}\text { Transmission } \\
\text { Hz }\end{array}$ & $\begin{array}{c}\text { SD } \\
\text { m }\end{array}$ & $\begin{array}{c}\text { SR } \\
\text { m }\end{array}$ & $\begin{array}{c}\text { RD } \\
\text { m }\end{array}$ \\
\hline \hline 108 & $09: 43$ & CW 115 & 39 & 535 & $50-60$ \\
\hline 109 & $09: 48$ & CW 150 & 39 & $"$ & $"$ \\
\hline 110 & $09: 54$ & CW 175 & 40 & $n$ & $"$ \\
\hline 111 & $09: 59$ & CW 200 & 39 & $n$ & $n$ \\
\hline 207 & $11: 19$ & CW 100 & 40 & $n$ & $50-55$ \\
\hline 208 & $11: 24$ & CW 100 & 40 & $n$ & $n$ \\
\hline 209 & $11: 29$ & CW 100 & 40 & $n$ & $n$ \\
\hline
\end{tabular}

$\mathrm{CW}$ : continuous wave at specified frequency. SD: source depth. SR: sourcefirst receiver range. RD: first receiver depth.

multiple hydrophone fit (neglecting array deformations along the horizontal plane), giving a stable source-first receiver range estimated of approximately $535 \mathrm{~m}$.

An obvious limitation of our experimental equipment is that it was not possible to acquire source-receiver distance information at the same time as the $\mathrm{CW}$ acoustic transmission. However, since the estimated source-receiver distance is very stable, it has been assumed that it remained constant at $535 \mathrm{~m}$ during the $\mathrm{CW}$ transmission.

\section{B. Ground Truth Identification}

In order to assess the potential of the proposed methodology, independent geoacoustic measurements have been performed, on different days, on the same track as the acoustic data. One of the problems in obtaining independent measurements is that it is necessary to use the same techniques that exhibit all the drawbacks that the proposed acoustic method wants to avoid.
TABLE II

Measured Geophysical Properties of Cores 280 and 281

\begin{tabular}{|c|c|c|c|c|c|}
\hline $\begin{array}{c}\text { Core } \\
\#\end{array}$ & $\begin{array}{c}\text { P vel } \\
\mathrm{m} / \mathrm{s}\end{array}$ & $\begin{array}{c}\text { Grain sz } \\
(\text { mean) } \phi\end{array}$ & $\begin{array}{c}\text { Porosity } \\
\%\end{array}$ & $\begin{array}{c}\text { Density } \\
\text { (wet) } \mathrm{g} / \mathrm{cm}^{3}\end{array}$ & $\begin{array}{c}\text { Carbonate content } \\
\%\end{array}$ \\
\hline 280 & 1546 & 6.49 & 62.7 & 1.63 & 73.9 \\
\hline 281 & 1562 & 6.61 & 70.8 & 1.54 & 72.7 \\
\hline 281 & 1593 & 3.92 & 57.2 & 1.72 & 87.9 \\
\hline
\end{tabular}

The first two readings refer to a depth of $0.1 \mathrm{~m}$ within the sediment. The last reading refers to a depth of $1.1 \mathrm{~m}$ within the sediment for core no. 281 . No such reading was possible for core no. 280 .

The following procedures were adopted.

- Seismic Survey: Conducted by simultaneously towing, over the same track, a $3.5-\mathrm{kHz}$ boomer and a $5000 \mathrm{~J}$ sparker. It provides a qualitative image of the seafloor and of the fine layering structures (Fig. 4). Note that during this survey the ship moved in the opposite direction from the towed array track.

- Coring: Two gravity cores (labeled as core no. 280 and core no. 281) were taken along the track; however, with very poor penetration. The cores were analyzed in the laboratory once back from the cruise. From the core measurements the surficial sediment properties used in the determination of the ground truth model are reported in Table II. In Table II the measurements for core 280 were made at a depth of $0.10 \mathrm{~m}$ within the sediment. For core 281 two sets of measurements at depths of 0.10 and $1.10 \mathrm{~m}$ were obtained. As a general description, the bottom in the area can be considered as mostly composed by carbonate sand, with a thin cover of sandy silt [6].

- Seismic Interface Waves: An array of geophones was deployed at the seafloor to measure the propagation of 
TABLE III

Interval Shear Wave Speed Estmated from the Disperston Characteristic of Seismic InTerface WaVes

\begin{tabular}{|c|c|}
\hline $\begin{array}{c}\text { Depth below seafloor } \\
\text { m }\end{array}$ & $\begin{array}{c}\text { S vel } \\
\mathrm{m} / \mathrm{s}\end{array}$ \\
\hline $0-2$ & 225 \\
\hline $2-4$ & 229 \\
\hline $4-6$ & 247 \\
\hline $6-8$ & 274 \\
\hline $8-10$ & 292 \\
\hline $10-12$ & 294 \\
\hline $12-15$ & 287 \\
\hline $15-18$ & 299 \\
\hline$\geq 18$ & 358 \\
\hline
\end{tabular}

TABLE IV

Geoacoustic Model Obtained From Independent Measurements aNd HaMILTON'S REgRESSION CuRves at tHE CORE No. 280 Site

\begin{tabular}{|c|c|c|c|c|c|c|}
\hline $\begin{array}{c}\text { Depth } \\
\mathrm{m}\end{array}$ & $\begin{array}{c}\mathbf{P} \text { vel } \\
\mathrm{m} / \mathrm{s}\end{array}$ & $\begin{array}{c}\mathbf{S} \text { vel } \\
\mathrm{m} / \mathrm{s}\end{array}$ & $\begin{array}{c}\mathbf{P} \text { att } \\
\mathrm{dB} / \lambda\end{array}$ & $\begin{array}{c}\text { S att } \\
\mathrm{dB} / \lambda\end{array}$ & $\begin{array}{c}\text { Dens } \\
\mathrm{g} / \mathrm{cm}^{3}\end{array}$ & Description \\
\hline \hline 0 & 1507 & 0 & 0 & 0 & $\overline{1}$ & water column \\
\hline 11.8 & 1510 & 0 & 0 & 0 & 1 & \\
\hline $118-124$ & 1550 & 230 & 0.17 & 3 & $\mathrm{~L} .63$ & recent sediments-sand \\
\hline $124-126$ & 1585 & 275 & 0.17 & 3 & 1.7 & transition-sand \\
\hline $126-136$ & 1610 & 290 & 0.4 & 3.3 & 1.7 & quaternary sediments - sand \\
\hline$\geq 136$ & 1700 & 360 & 0.37 & 3.3 & 1.82 & quaternary sedimenis - sand \\
\hline
\end{tabular}

Depth is assumed to be $0 \mathrm{~m}$ at the water surface.

seismic interface waves in situ. The array was deployed at approximately the same position as core no. 280, along the acoustic track direction. From the dispersion of the interface waves, a model of shear wave speed as a function of depth for the first fifteen meters of the bottom was obtained according to the procedures of Caiti et al. [7]. The results are reported in Table III.

In order to produce a ground truth geoacoustic model, the above-mentioned measurements have been used with Hamilton's correlation tables and regression curves [8]. In particular, we firstly computed a compressional wave speed profile as a function of depth from the shear wave speed profile and the surficial measurement of core no. 280 , using the equations in [8, p. 31].

The ground truth Hamiltonian model thus obtained is reported in Table IV, where the depth referred to is at the location of core no. 280. Since this is very local information it was assumed that these geoacoustic properties remained constant along each bottom layer of the seismic survey track shown on Fig. 4. Therefore, the towed array bottom inversion will be evaluated against information of Table IV extrapolated, for each layer, along the layering structure of Fig. 4.

\section{Nonacoustic Sensor Positioning Data}

The sensor positioning data was obtained from six recording modules, each one composed of one tiltmeter, one compass, and one accelerometer located in the towed array, as shown in Fig. 5. In the head and tail modules there are also pressure gauges for depth monitoring. All the sensor positioning system information was cabled to the two ship, digitized, processed, and displayed in real time. The procedure used was simply based on recording the horizontal (compass) and vertical (tiltmeter) deviations from a reference hydrophone,

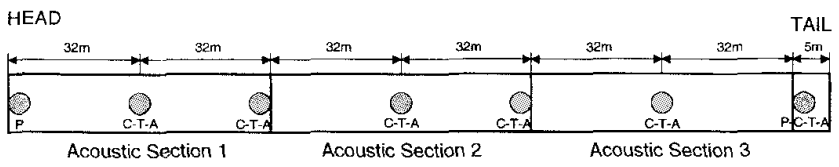

Fig. 5. Towed array configuration and sensor positioning modules: $C=$ compasses, $T=$ tiltmeters, $A=$ accelerometers, and $P=$ pressure gauges.

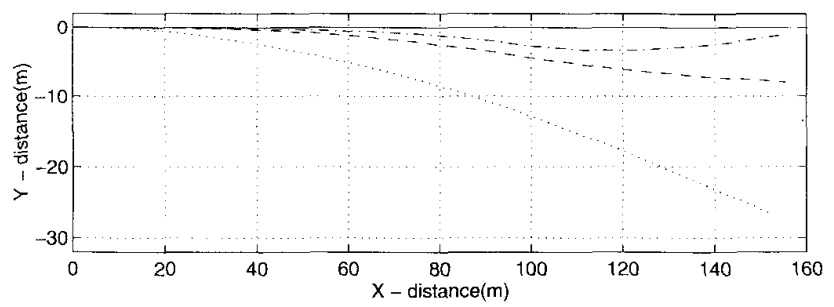

(a)

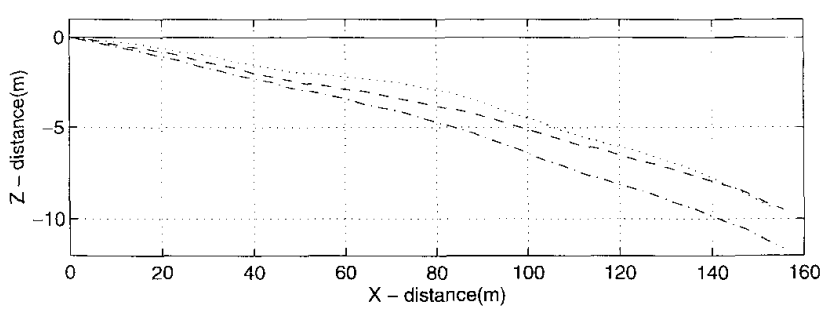

(b)

Fig. 6. Array shape projections on (a) horizontal plane $X-Y$ and on (b) vertical plane $X-Z, X$ axis is oriented along ship track: straight line for reference $(-$ solid $)$, before the turn $(\cdots$ dash-dot $)$, during the turn $(\cdots$ dotted $)$ and after the turn (-- dashed).

and on a polynomial interpolation scheme to obtain the shape deformation between the module positions. The accelerometer information was found redundant, very noisy, and finally not used for estimating array shape deformations. As an example, Fig. 6 shows the at sea recorded array deformations before, during, and after a $180^{\circ}$ ship turn. It can be noted that, in general, the array is never straight or horizontal even when the ship is steaming along a straight line.

Using the time information, the estimated array shape deformation is correlated to the acoustic field snapshots, so that each snapshot during a run is associated to a set of hydrophone positions. Fig. 7 shows a three-dimensional perspective of the estimated array deformation during a 5-min interval (run 209 in this example). Strong depth variations can be observed that have to be taken into account in the inversion since they represent deviations of up to and more than a wavelength at the highest frequency considered.

It has been observed [9] that, using the $X-Y$ plane estimated positions on $360^{\circ}$ planewave beamforming, ship echoes remain coherent throughout the turn and can be unambigously resolved from the left and right sides of the ship track corresponding to their true locations. These observations do not mean that the hydrophone positions have been exactly estimated but assert that the position estimates are consistent with the ship movement and, according to the observed mainlobe degradation, at least within $\lambda / 2$ of the true position (on the $X-Y$ plane), where $\lambda$ is the acoustic wavelength. 


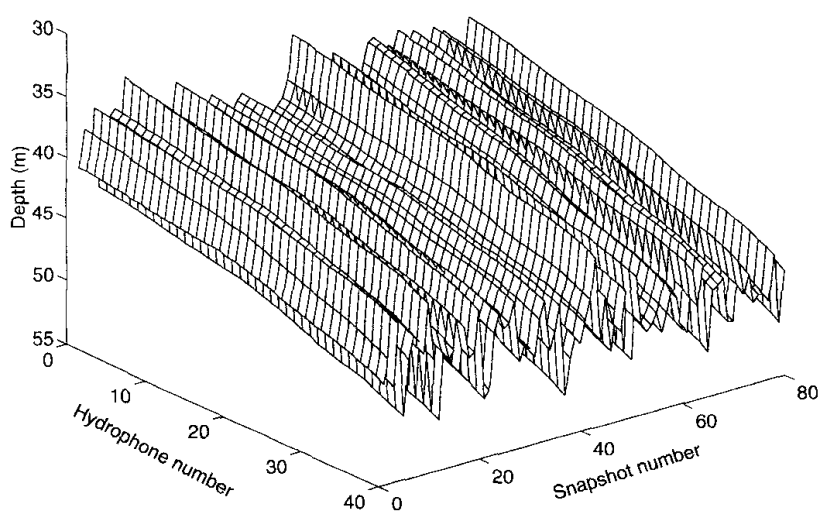

Fig. 7. Array shape deformation versus snapshot number for run 209 .

\section{DATA INVERSION AND SEAFLOOR MODEL IDENTIFICATION}

The data used for the estimation of the seafloor parameters are the acoustic field snapshots obtained from the CW signals transmitted as described in Section II. By inverting each individual snapshot, one gets a series of estimated geoacoustic parameters as a function of time along the surveyed track. One well-known character of the data inversion is that it is a strongly nonlinear optimization problem [5, pp. 109-114] moreover, since an inverse problem has to be solved for each snapshot, the computation of the inverse solution over all the runs becomes very demanding from a numerical point of view.

To deal more efficiently with the computational complexity of the problem, we have tested two alternative methods. In the first, a matched-field-like objective function is maximized with a genetic algorithm (GA). In the second, a regular approximation of the nonlinear inverse function is determined as a series of radial basis functions (RBF).

Both methods have already been discussed in general elsewhere [5, pp. 177-182], [10], [11], and here we focus on the description of how both techniques are able to reduce the computational effort, and on the computational cost in terms of accuracy of the estimated solution.

Both methods use the SAFARI/OASES code [12] as the numerical engine for the computation of the forward problem. There is also an important difference in the two algorithms that we wish to mention at this point: the GA algorithm uses both amplitude and phase of the received field, while the RBF algorithm uses only the amplitude information.

\section{A. Data Inversion with GA}

I) Background: This method starts from a very classical parameter estimation problem where the observation is a nonlinear, but (assumed) known function of the parameter vector corrupted by uncorrelated zero mean additive noise. Let $\boldsymbol{Y}=\left\{\boldsymbol{y}_{n} ; n=1, \cdots, N\right\}$ be the observation ensemble with $\boldsymbol{y}_{n}$ being an $L$ multivariate normally distributed complex random variable representing the snapshot $n$ of the received acoustic pressure and modeled by

$$
\boldsymbol{y}_{n}\left(\omega_{k}, \gamma_{T}\right)=b_{n}\left(\omega_{k}\right) \boldsymbol{p}\left(\omega_{k}, \gamma_{T}\right)+\boldsymbol{\epsilon}_{n}\left(\omega_{k}\right), \quad k=1, \cdots, K
$$

representing the nondeterministic source amplitude at the receiver due to environment fluctuations not included in the noise process $\boldsymbol{\epsilon}_{n}$, and $\boldsymbol{p}\left(\omega_{k}, \gamma_{T}\right)$ is the $L$ sensor vector solution of the wave equation at frequency $\omega_{k}$ for the true parameter vector $\gamma_{T}$. An estimator $\hat{\gamma}_{T}$ of $\gamma_{T}$ given $Y$ is [10]

$$
\hat{\gamma}_{T}=\arg \left[\max _{\gamma}\left\{\boldsymbol{p}\left(\omega_{k}, \gamma\right)^{H} \hat{\boldsymbol{R}}_{y}\left(\omega_{k}\right) \boldsymbol{p}\left(\omega_{k}, \gamma\right)\right\}\right]
$$

where $\hat{\boldsymbol{R}}_{y}\left(\omega_{k}\right)$ is the data sample covariance matrix at frequency $\omega_{k}$ given by the average of the data outer products, $N \hat{\boldsymbol{R}}_{y}=\boldsymbol{Y} \boldsymbol{Y}^{H}$. We fixed $N=1$ (i.e., we inverted one single snapshot at a time), and adopted a GA-like strategy for solving the optimization problem of (2) [13]. In order to speed up the standard GA, we have introduced some modifications, the most important of which consists in progressively reducing the parameter search space during the GA search. The price paid for the speed up in computation is an increased probability of getting stuck in a local maximum of the fitness function, the very problem global search algorithms try to avoid. A more extensive description of the modifications introduced in the basic GA, together with some examples, can be found in [12]. Here the GA parameters were chosen as follows: the population size was set to 30 , the reproduction size was 0.5 , the crossover probability was 0.8 and the mutation rate was set of 0.05 . The total number of forward model runs was limited to 1500. As shown in [5, pp. 109-114], an important requirement for reaching an unbiased estimate is the adequate specification of the environmental model (water depth, layer thickness, etc.) and of the receiving geometry: source-receiver range, relative depths, etc. One way to achieve this is to include layer thickness and water depth in the search space with relatively narrow variation intervals around the expected values allowing for a better fit [higher fitness function (2)] and convergence to an unbiased parameter vector estimate. This procedure of including "known parameters" into the search is called focalization and it has been proved successful, for instance, in [14] and [15]. Although, focalization techniques increase the number of parameters to be estimated, and also increase the amount of computation required by the search algorithm. In trying to limit this increase in computational cost while still preserving the benefits of focalization, we have adopted a double-step strategy that is described in the next subsection.

2) Data Inversion Procedure: The data inversion procedure was separated into two parts: first, the model and geometry setting, and then the inversion of the sequence of snapshots. During model setting a single acoustic field snapshot was selected for each run (typically at the beginning of the run) and the GA inversion attempted for compressional velocity, water depth, and layer thickness. That is, focalization was employed during model setting, increasing the required number of forward model runs. The estimated layer thicknesses and water depth was then held constant for the inversion of all other snapshots within the same run (approx. $5 \mathrm{~min}$ ). In the course of GA inversion, the array position estimated from the nonacoustic sensor was used to specify the receiver depths. The GA parameters for the inversion of all the snapshots have been reported in the previous subsection. Note that, with 
our parameter setting, the inversion of a single run along the track requires approximately 150000 forward model runs. The inversion results with this method have been found to be very insensitive to shear speed, density and attenuation, and no significant results for these parameters have been obtained.

\section{B. Data Inversion with Radial Basis Functions ( $R B F$ )}

The RBF approach is based on the idea of approximating the inverse function through a series of basis functions belonging to the RBF class. So, each seafloor parameter $m_{i}$ of interest is estimated through the following expression:

$$
m_{i}=\sum_{j=1}^{N} c_{i j} \phi\left(\left\|x-x_{j}\right\|\right)
$$

where $\phi(r)$ is a specific RBF, and $\boldsymbol{x}$ is the vector of acoustic data to be inverted. We remark that the data vector $\boldsymbol{x}$ used with the RBF algorithm is the square amplitude of the acoustic field snapshot, expressed in decibels (i.e., the received power at the transmitting frequency).

The coefficients $c_{i j}$ and the centers $x_{j}, j=1, \cdots, N$, are determined in the following way. Each $\boldsymbol{x}_{j}$ is the acoustic field corresponding to a known seafloor model, computed with SAFARI, with all the geometric and environmental information (i.e., source-receiver distance, receiver positions, sound speed profile in the water, water depth,...) corresponding to those of the experimental situation. In practice the centers $x_{j}$ play the role of points in the space of the possible acoustic measurements for which the inverse solution is known. The whole inverse approximation is then built by interpolating through the known values with functions having radial symmetry. The reader is referred to [16] for a general discussion on the properties of such an approximation. For the purpose of the present paper it is sufficient to say that, among the possible choices of RBF, we have employed Gaussian functions (i.e., $\phi(r)=\exp \left(r^{2} / \sigma^{2}\right)$ ) in order to enforce the regularity properties of the approximated inverse. The RBF approximation will be convergent to the true solution as the number of centers $N$ approaches infinity. As shown in [5, pp. 177-182], [6], [11], it is possible to achieve an expected accuracy of about $1 \%$ for the compressional velocity and $5 \%-10 \%$ for the shear velocity by using a number of centers of the order of 900 . The centers are generated by randomly picking a physically meaningful seafloor model and computing the corresponding acoustic field. The set of centers thus generated is referred to as the "training set" (from the neural network jargon) and it is employed also to determine the coefficients $c_{i j}$ by evaluating the RBF expansion in the known points and generating a system of linear equations that is solved with standard techniques in the $c_{i j}$ unknowns.

It is important to underline at this point that the RBF method does require the precise knowledge of all the geometric parameters before the generation of the training set. On the other hand, if several data sets share the same geometric configuration, the same RBF expansion can be used to invert these data sets without the need of computing another training set. Another important point is that, at least in our current implementation, we are not able to deal with layers of variable thickness in an efficient way, while the insertion of additional layers poses no major problems. So our implementation decision has been to fix a priori the layer thickness and the number of layers. This has been done by considering, for any given frequency $\omega$, the maximum penetration, $z_{w}=\lambda$, and the maximum resolution, $h_{\omega}=\lambda / 3$, expected at that frequency. The layer thickness is then fixed as $h_{\omega}$, and the number of layers as $z_{\omega} / h_{\omega}$. For modeling purposes, the last layer is treated as an infinite half-space, however, the results are not considered valid beyond the $z_{\omega}$ depth. Note that this is a "rule of thumb," in the sense that we have found in simulations [5, pp. 177-182], [6] that the method is in general very poorly sensitive to seafloor parameters below the cutoff depth $z_{\omega}$, and it is not able to resolve layers of thickness smaller than $h_{\omega}$. Of course, caution must be taken in generalizing this rule: a hard rock layer placed at a depth just below $h_{\omega}$ will have a strong effect on the acoustic field and in the inversion result; however, this is not the case for the data discussed here.

In the remaining subsections we will discuss the following specific topics: clustering of the array shape geometric data, smoothing of the acoustic data before the inversion, and uncertainty estimate of the inversion results.

1) Geometric Data Clustering: The RBF scheme is efficient if the same approximating function can be employed to invert several data vectors without the need of generating a new training set. However, a training set is associated specifically with a given geometrical configuration. The movement of the array under tow, as reported in Fig. 7, precludes the use of the same geometrical information for all the data sets of the same run, even when the water depth and the sound speed profile in the water are constant. However, the movement does have some periodicity, so that the same position is repeated after some time interval. We have employed a standard clustering algorithm to automatically separate all the data sets of the same run into classes of data homogeneous with respect to the geometric position. In particular, we have associated with the same cluster all the geometric positions that did not differ from each other in depth by more than one meter (for any of the hydrophone positions). Once the data have been divided into different clusters, each cluster is characterized by a mean receiver array position computed by averaging for each hydrophone the position of the elements belonging to the cluster.

By using the averaged positions, an RBF inverse function can be determined for each cluster, and the data belonging to the same cluster are processed by the same RBF inverse. With the data gathered in the experiment, it has been possible to divide roughly every run of 100 snapshots in five homogeneous clusters. This means that, knowing that 900 forward model runs were used for the computation of the RBF inverse of each of the five clusters, a total number of 4500 model runs were needed to invert each data run of 100 snapshots.

Note also that, in the computation of the training sets for each run, the water depth has been maintained constant. In the runs from 108 to 111 , where the water depth was slowly varying, the mean water depth for each run has been used. This introduces another approximating factor in the inversion (hence a potential source of error). 


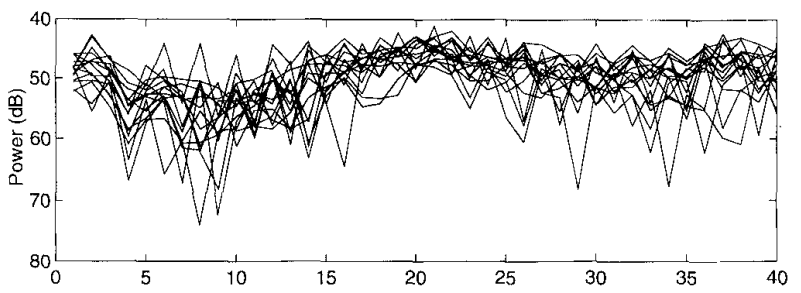

(a)

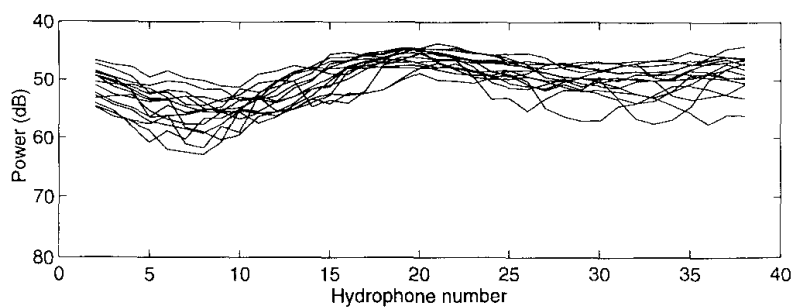

(b)

Fig. 8. Several snapshots of array received power for run 209 at $100 \mathrm{~Hz}$; (a) directly measured and (b) after 4-point average smoothing.

2) Acoustic Data Smoothing: Once the data are divided into different clusters on the basis of the geometric position of the receivers, a training set for each cluster can be generated. The acoustic field snapshots corresponding to the geometric position belonging to the cluster are then given as input to the RBF inverse and the estimated seafloor parameters are obtained. Once the data of all the clusters have been inverted, the data are rearranged in sequence according to the time at which each snapshot has been extracted.

One of the problems encountered in the experiment has to do with the data variability. In particular, in Fig. 8(a) the acoustic field snapshots belonging to the same cluster are reported. Note that the data in each cluster are not taken at consecutive time intervals, but share the same receiver depths. It can be seen that the data display a high level of variability, that cannot be solely due to the variability in the seafloor parameters and/or the fluctuations in the transmission medium. In order to reduce the noise, a 4-point moving average has been applied to the data, leading to a more stable behavior of the field, while still preserving its structure. In Fig. 8(b) the filtered data are shown.

3) Uncertainty Estimation: Once the data in each cluster have been inverted, all the data are reorganized according to the time information, and arranged as a function of time along the track. It has to be considered that the inversion results are not due to the seafloor structure at a single point, but the acoustic data are receiving energy from an extended region of the seafloor. From the geometry of the experiment, we estimated this region to roughly be $75 \mathrm{~m}$ in range along the track. To take this into account in the resulting display, a moving average of the inversion results from each snapshot is performed, with a window of 12 snapshots, corresponding to $36 \mathrm{~s}$ of data. Once the moving average has been computed, the standard deviation with respect to the computed average is calculated, and displayed together with the estimated parameters. This allows us to give a measurement of the uncertainty in the inversion results, and to assess their reliability.

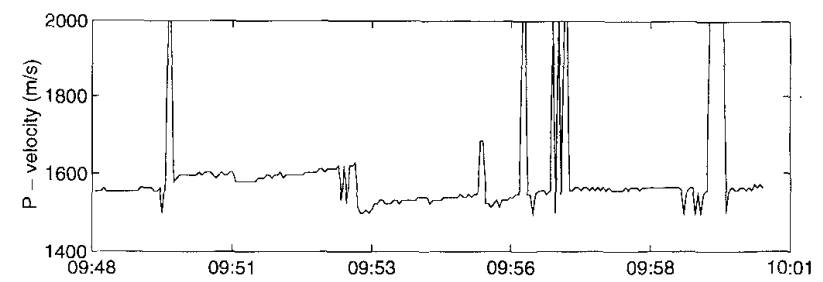

(a)

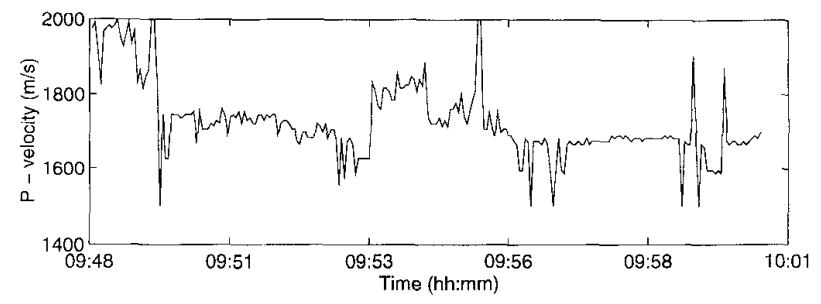

(b)

Fig. 9. Genetic Algorithms inversion results of P-velocity for runs 109, 110 , and 111: (a) first bottom layer, 15-m thick and (b) second bottom layer, $5-\mathrm{m}$ thick.

TABLE $V$

Ga Estimated Mean and Standard Deviations of COMPREssional Velocities for the First Two Layers

\begin{tabular}{||c|c|c|c|c||}
\hline RUN & \multicolumn{2}{|c|}{ Layer 1 } & \multicolumn{2}{c||}{ Layer 2 } \\
mean & $\begin{array}{c}\text { std. } \\
(\mathrm{m} / \mathrm{s})\end{array}$ & $(\mathrm{m} / \mathrm{s})$ & $(\mathrm{m} / \mathrm{s})$ & $(\mathrm{m} / \mathrm{s})$ \\
\hline 109 & 1591 & 66 & 1730 & 113 \\
\hline 110 & 1532 & 33 & 1794 & 65 \\
\hline 111 & 1557 & 147 & 1675 & 56 \\
\hline
\end{tabular}

Thickness of layer 1 estimate was $15 \mathrm{~m}$. Thickness of layer 2 estimate was $5 \mathrm{~m}$

\section{RESUlts DISCUSSION}

There are several points that have to be addressed in order to properly discuss the merits and drawbacks of the methodology proposed. In the following, we first compare the towed array results with the ground truth Hamilton-like seafloor model, then we compare the results obtained with RBF and GA, and comment on their relative precision and computational effort.

\section{A. Inverse Model and Ground Truth}

The GA data inversion results are shown in Table $\mathrm{V}$ and depicted in Fig. 9. The inversion procedure described in Section IV-A-2 gave layer thicknesses estimates of $15 \mathrm{~m}$ for the first layer and $5 \mathrm{~m}$ for the second layer. These values roughly agree with the layering of Fig. 4 close to Core 281 location, layer $\mathrm{A}$ is $15 \mathrm{~m}$ thick, layer $\mathrm{A} / \mathrm{B}$ is $5 \mathrm{~m}$ thick, and layer B was not reached.

It can be remarked that the mean compressional velocities are in good agreement with those found by direct measurements and also (see below) with RBF results on the same portion of the track. It can also be noted that there is a slight tendency to over estimate the velocities, especially in the deeper layer. This may be due to the transition zone, denoted $\mathrm{A} / \mathrm{B}$ in Fig. 4 , and not present near Core 280. Fig. 9 shows snapshot-to-snapshot inversion results between 09:48 and 10:05. It can be noted that at several instants the GA did 

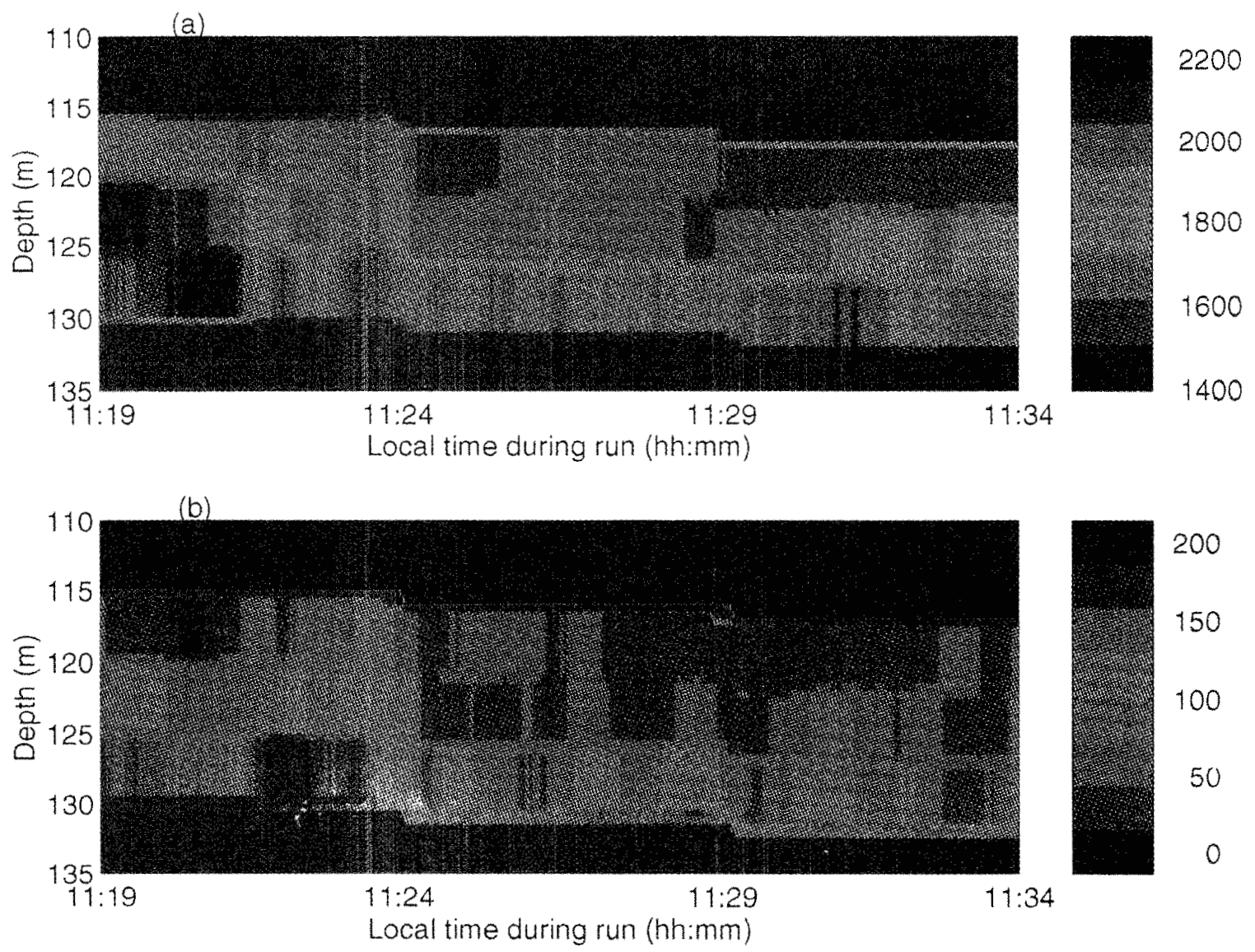

Fig. 10. Radial Basis Functions results for runs 207, 208, and 209: (a) estimated P-velocity, (b) respective estimated uncertainty. Dark blue represents the water, red line represents seafloor interface, $\mathrm{P}$-velocity into the bottom is color coded in $\mathrm{m} / \mathrm{s}$ and dark pink is noninverted bottom below the sediment.

not converge and the estimate was found at the extreme of the search interval. In general, the variability is, as expected, much higher for the second layer than for the first layer.

The results of the RBF inversion along the two portions of the survey track are reported in Figs. 10-13. Note that the part of the track closest to the position of core no. 280 (and so directly comparable with the ground truth model of Table II) is in Figs. 10 and 11. It can be noted that the uncertainty figures show that the estimated P-velocity reliability decreases with depth (P-velocity is better determined in the upper portion of the sediment), while S-velocity has a large uncertainty in the uppermost part, and the uncertainty decreases with depth. This is in accordance with the relative influence on the acoustic field that may be expected for $\mathrm{P}$ - and $\mathrm{S}$-velocities in unconsolidated sediments at the frequencies transmitted [17]. It has also to be mentioned that attempts at estimating attenuations and density totally failed, producing very large uncertainties over the entire track.

The first comment is that there is indeed a good qualitative agreement between the ground truth model and the inversion results. There is also quantitative agreement for the first 10 $\mathrm{m}$ within the sediment, but in the $10-15 \mathrm{~m}$ depth region the compressional velocity is consistently estimated higher than the ground truth value in the case of RBF inversion (about
$1850 \mathrm{~m} / \mathrm{s}$, versus $1610-1700 \mathrm{~m} / \mathrm{s}$ ). This difference cannot be accounted for by the uncertainties in the RBF approximation only. However, it is not easy to establish the cause; while the RBF high velocity estimate is consistent along the track, the ground truth model at that depth has been obtained just by application of Hamilton's curves. A previous experiment in the Adventure Bank area has shown, at least for shear waves, that the in situ velocity is closer to the spread in Hamilton's regression curves for gravel than to that for coarse sand [7]. Whether this may be due to diagenetic phenomena linked with the rich content of calcium carbonate found in this area we are not able to assess.

The conclusion is that there is a consistent quantitative agreement in the results obtained in the first $10 \mathrm{~m}$ of sediment, while there is a systematic difference between the ground truth model and the RBF inversion results in the $10-15 \mathrm{~m}$ depth region. This difference does not change the qualitative character of both models, that are still in agreement, but we are not able to account for it with the data presented.

\section{B. Comparison Between $G A$ and $R B F$}

An important consideration at this point is that the obtained inverse model is richer, in information, than the ground truth reference model. In particular, it is possible to see some lateral 

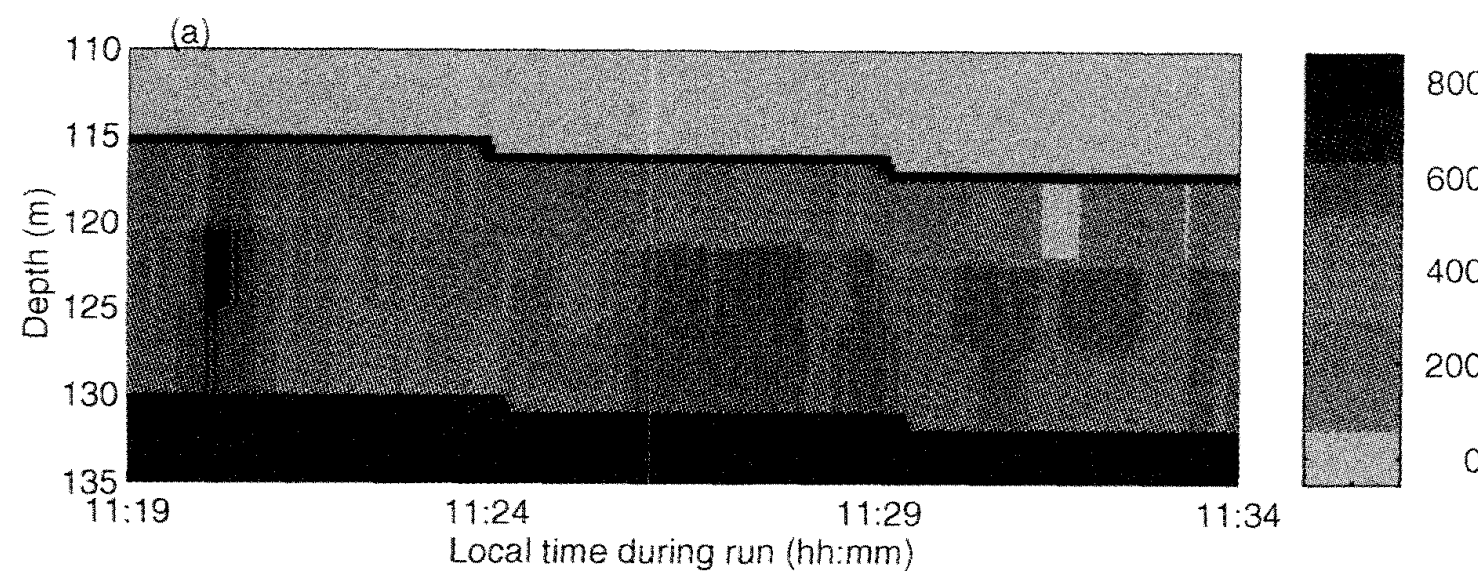

600

400

200
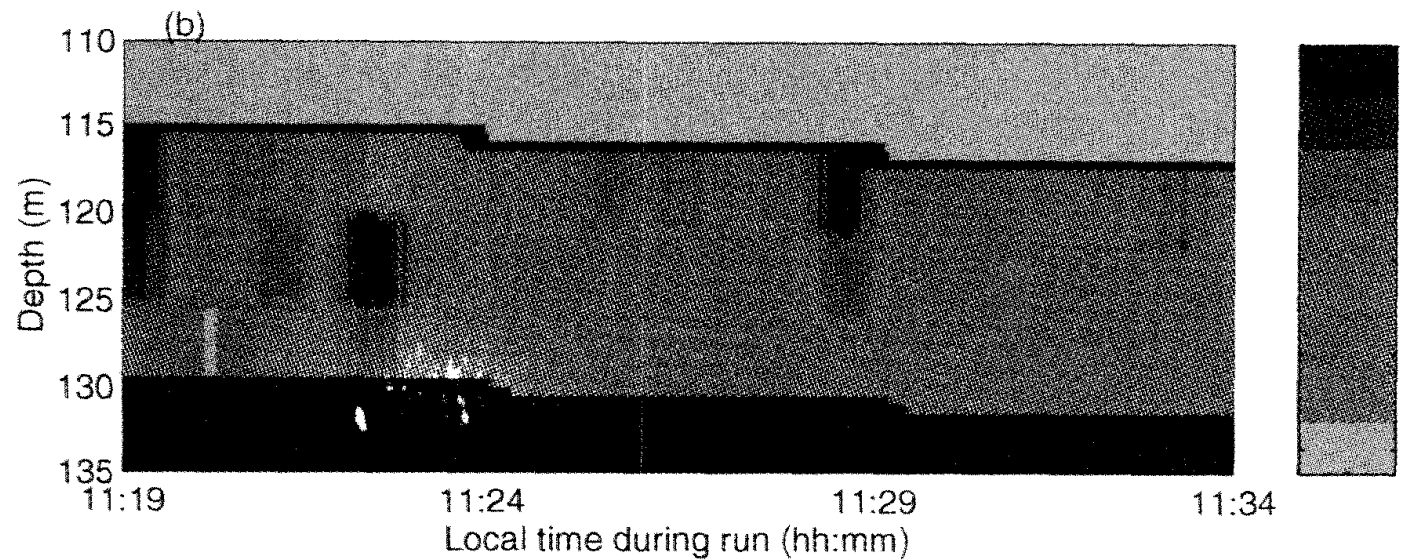

200

150

100

50

0

Fig. 11. Radial Basis Functions results for runs 207, 208, and 209: (a) estimated S-velocity, (b) respective estimate uncertainty. Dark blue represents the water, red line represents seafloor interface, S-velocity into the bottom is color coded in $\mathrm{m} / \mathrm{s}$ and dark pink is noninverted bottom below the sediment.

variation of the seafloor properties that is not accounted for by the reference model. The reason is rather obvious; the reference model has been calibrated with in situ measurements at one point (that of core no. 280), and the measurements extrapolated over the whole track with the aid of the seismic charts. In order to account for lateral variation, the reference model should have been calibrated at several points; this could not be done in the experiment due to lack of ship time, and this is the very problem that the acoustic inversion attempts to overcome.

Both the RBF and GA inversion schemes provided qualitatively similar and consistent results. The GA results also compared well quantitatively, with the independent ground truth measurements. GA inversion could provide these results only for the higher frequency runs $(\geq 150 \mathrm{~Hz})$, while the RBF scheme worked in the whole frequency range with slightly more consistent results at lower frequencies $(\leq 125 \mathrm{~Hz})$. RBF allowed for the estimation of shear velocity (not everywhere, though), while the GA method was found completely insensitive to shear.

Note that there are basic differences in the two inversion algorithms that may account for the difference in the results. There is first of all the use of the data (GA uses both amplitude and phase information, while RBF uses only amplitude); then there is the difference in the approach (explicit maximization of a fitness function for GA, interpolation in a multiparameter space for RBF). For instance, it is likely that the better behavior of RBF at lower frequency is due to the fact that the acoustic field (as a function of range) is smoother at lower frequencies, favoring the interpolation algorithm. Note that the lower the frequency, the more sensitive is the acoustic field to shear properties. However, it is difficult to assess how the methodological differences in the inversion algorithms are exactly reflected in the observed results.

Knowledge of array deformation was found to be essential for inversion at wavelengths less then or equal to 1.5 times the depth difference between towed array head and tail. When the array shape is relatively constant, computational efficiency of RBF is many order of magnitude higher than that of GA, and in that case, real time estimation is possible with RBF.

\section{Conclusion and Future Trends}

This paper describes the experimental results obtained by trying to estimate a geoacoustic model of the bottom using a moderate aperture towed array in shallow water and inverting the received acoustic field with global search strategies. One crucial aspect of the method proposed is the monitoring in 

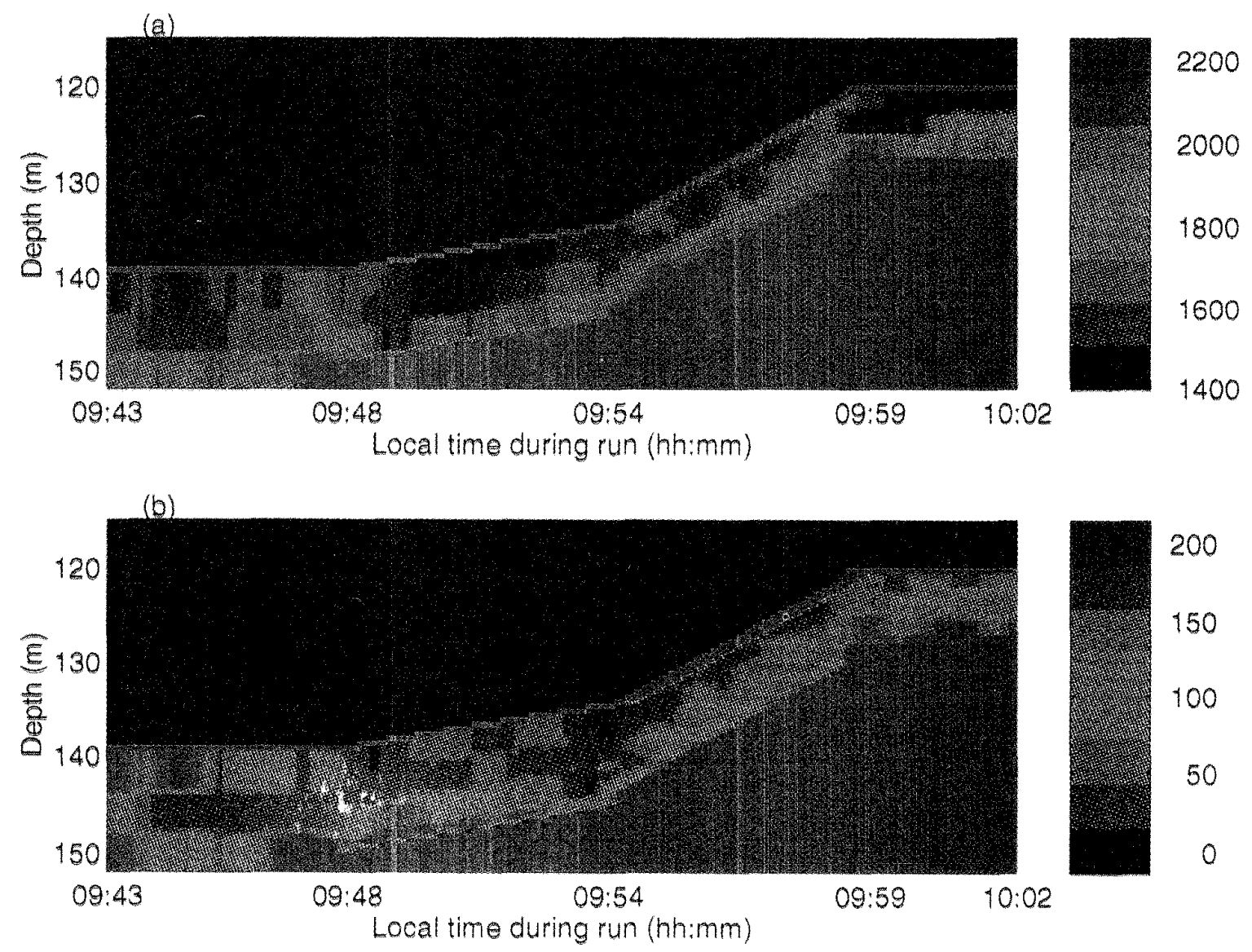

Fig. 12. Radial basis functions results for runs 108 to 111: (a) estimated P-velocity, (b) respective estimate uncertainty. Dark blue represents the water, red line represents seafloor interface, P-velocity into the bottom is color coded in $\mathrm{m} / \mathrm{s}$ and dark pink is noninverted bottom below the sediment.

real time of the receiving array shape. The results obtained are in good qualitative and, up to a certain extent, quantitative agreement with an independent seafloor reference model obtained by combining in situ measurement with Hamilton's tabulations. Moreover, the results of the acoustic inversion are much richer in information than those of the reference model and, therefore, not easily comparable. Further investigation is certainly needed, however, the overall agreement found so far suggests that the proposed method is capable of overcoming some of the limitations of the traditional techniques.

We consider the experiment described in this work as a feasibility test of the whole concept of geoacoustic model identification with towed array acoustic data. As a feasibility test, we can safely state that it can be considered successful. However, we are aware that several improvements are certainly needed to obtain better performance in future operations with the same instrumentation. We would like at this point to briefly mention those improvements on which we are currently working.

- Array Motion Estimation: It is clear that the correct monitoring of the array shape is critical to the success of the method. The motion monitoring has been obtained by interpolating the measurements of the nonacoustic sensors. One possible way to improve the monitoring of the array shape is to couple the measurements with a dynamic model of the array motion, as in [18].

- Data Inversion: We have been concentrating on finding self-contained methods that can efficiently solve the inverse problem. As already discussed, the methods employed were specifically chosen to obtain a global solution while limiting the computations required. As a matter of fact, this means trying to achieve a tradeoff between accuracy in the solution and computational cost of the algorithm. Different inversion strategies may be considered. One possibility is to use a global search method at the beginning of the track. The model thus obtained can be used as the background model when inverting for the subsequent data, using a local method in the fashion of [3]. When the variation from the background model starts to become greater than a certain amount (using a prespecified measure), a new global search can be started, to recalibrate the local method. In this way, a combination of global and local methods would be obtained for the inversion of the whole acoustic track. Note that local methods are also able to provide an uncertainty measure for the estimated model.

- Resolution and Penetration Within the Bottom: Both resolution and penetration depend on the source frequency 

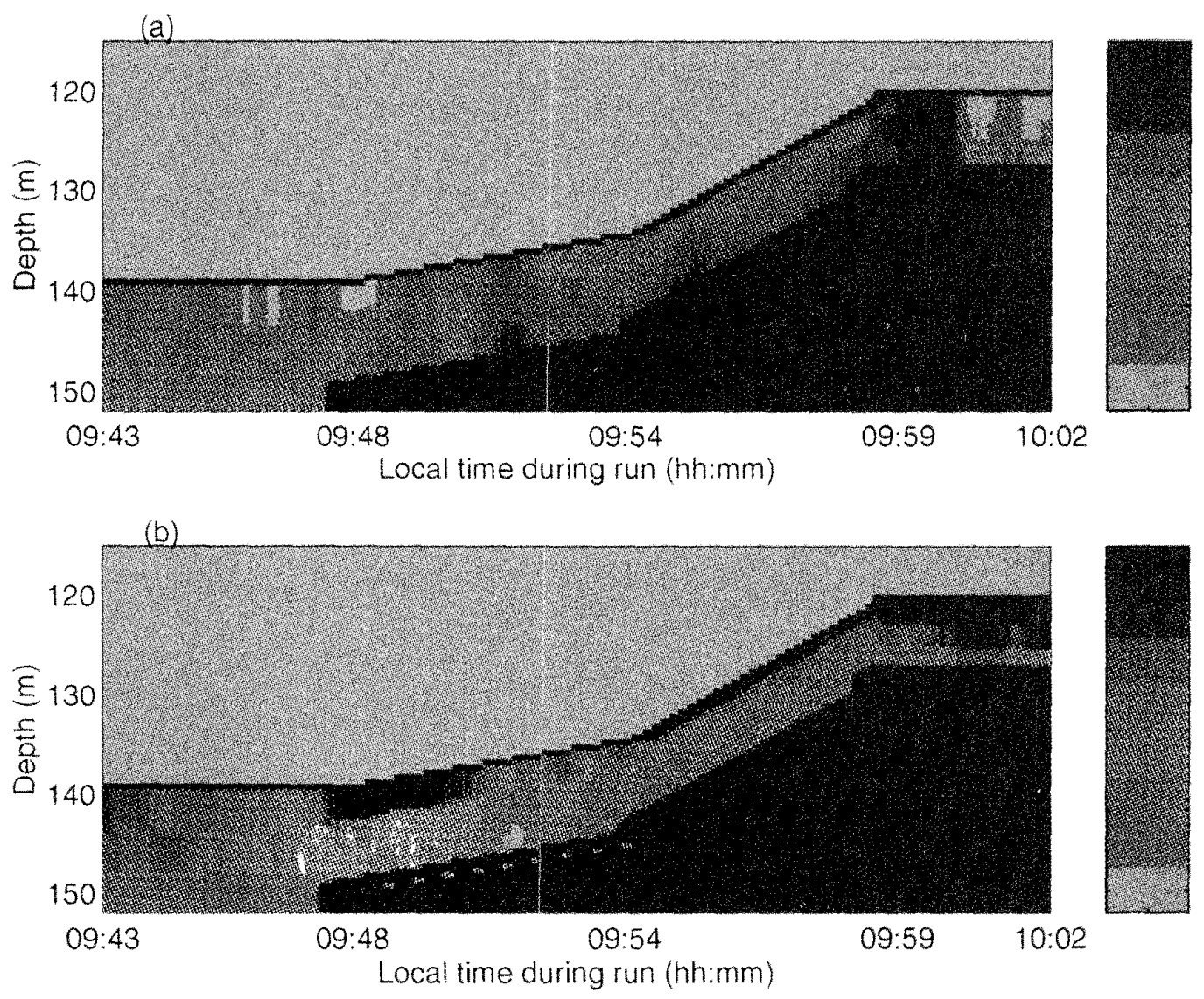

Fig. 13. Radial basis functions results for runs 108 to 111: (a) estimated S-velocity, (b) respective estimate uncertainty. Dark blue represents the water, red line represents seafloor interface, $S$-velocity into the bottom is color coded in $\mathrm{m} / \mathrm{s}$ and dark pink is noninverted bottom below the sediment.

transmitted. In our results of Figs. 9,12 , and 13, the penetration slightly increases (while the resolution decreases) accordingly with the changes in frequency during the experiment. The transmission of multiple frequencies simultaneously (say, for instance, ten tones from 50 to $500 \mathrm{~Hz}$ ) may help in increasing both penetration and resolution at the same time, and also in obtaining a more robust geoacoustic estimate.

\section{ACKNOWLEDGMENT}

The authors wish to acknowledge the master and crew of the R/V ALLIANCE and the SACLANT Centre Engineering Department for their outstanding respective contributions in the leadership, sea-going operation and equipment preparation before and during the sea trial. The support of E. Dias and E. Coelho from the Hydrographic Institute, Lisbon, on the acquisition of the nonacoustic data and of P. Gershoft, SACLANT Centre, on genetic algorithms setup, are also appreciated. The authors wish also to express their appreciation to the anonymous reviewers, whose comments have greatly helped to reshape the second draft of this paper, and hopefully to improve its readability.

\section{REFERENCES}

[1] G. V. Frisk, J. F. Lynch, "Shallow water waveguide characterization using the Hankel transform," J. Acoust. Soc. Amer., vol. 76, pp. 205-216, 1984.

[2] G. V. Frisk, J. F. Lynch, and J. A. Doutt, "The determination of geoacoustic models in shallow water," in Ocean Seismo-Acoustics, T. Akal and J. M. Berkson (Eds.), New York: Plenum, pp. 693-702, 1986.

[3] S. D. Rajan, J. F. Lynch, G. V. Frisk, "Perturbative inversion methods for obtaining bottom geoacoustic parameters in shallow water," J. Acoust. Soc. Amer., vol. 82, pp. 998-1017, 1987.

[4] M. D. Collins, W. A. Kuperman, H. Schmidt, "Nonlinear inversion for ocean-bottom properties," J. Acoust. Soc. Amer., vol. 92, pp. 2770-2783, 1992.

[5] Full-Field Inversion Methods in Ocean and Seismo-Acoustics, O. Diachok, A. Caiti, P. Gershoft, H. Schmidt (Eds.), Kluwer Acad., 1995.

[6] S. M. Jesus, R. Zoppoli, and H. Zambujo, "Geophysical seafloor exploration with a towed array in shallow water," 24-month report, Project MAS2-920022, DG XII/European Community, MAST II Programme, 1994.

17] A. Caiti, T. Akal, and R. D. Stoll, "Estimating of shear wave velocity in shallow marine sediments," IEEE J. Oceanic Eng., vol. 19, pp. 58-72, 1994.

[8] E. L. Hamilton, "Acoustic properties of sediments," in Acoustics and the Ocean Bottom, A. Lara-Saenz, C. Ranz-Guerra, L. Carbo-Fite (Eds.), Madrid, Spain, 3-58, 1987.

[9] P. Felisberto and S. M. Jesus, "Towed array beamforming during ship's maneuvering," Proc. Inst. Elect. Eng. on Radar, Sonar and Navigation, to be published.

[10] S. M. Jesus and A. Caiti, "Estimating geoacoustic bottom properties from towed array data," J. Comput. Acoust., to be published. 
[11] A. Caiti and S. M. Jesus, "Acoustic estimation of seafloor parameters: A Radial Basis Functions approach," J. Acoust. Soc. Amer., to be published.

[12] H. Schmidt, "SAFARI: Seismi-acoustic fast-field algorithm for range independent environments," SACLANTCEN Rep. SR-113, La Spezia, Italy, 1988. See also "OASES User's Guide," version 1.7 application and upgrade notes, available from the author.

[13] D. E. Goldberg, Genetic Algorithms in Search, Optimization and Machine Learning. New York: Addison-Wesley, 1988.

[14] M. D. Collins and W. A. Kuperman, "Focalication: Environmental focusing and source localization," J. Acoust. Soc. Amer., vol. 90, pp $1410-1422,1991$.

[15] D. F. Gingras and P. Gershoft, "Inversion for geometric and geoacoustic parameters in shallow water: experimental results," J. Acoust. Soc. Amer., vol. 97, pp. 3589-3598, 1995.

[16] T. Poggio and F. Girosi, "Networks for approximation and learning," Proc. IEEE, vol. 78, pp. 1481-1497, Sept. 1990.

[17] A. Kristensen and J. M. Hovem, "Sensitivity of bottom loss to attenuation and shear conversion," in Shear Waves in Marine Sediments, J. M. Hovem, M. D. Richardson, R. D. Stoll, (Eds.), Kluwer Acad., pp. 431-438, 1991.

[18] J. L. Riley and D. A. Gray, "Towed array shape estimation using Kalman filters-experimental investigation," IEEE J. Oceanic Eng., vol. 18, pp. 572-581, Oct. 1993

Andrea Caiti (M'92) received the "Laurea" degree in electronic engineering from the University of Genova, Italy, in 1988.

During 1989-94, he was a staff scientist at the SACLANT Undersea Research Centre, La Spezia, Italy. In 1994-95, he has been adjunct professor of System Analysis at DIST (Department Communication, Computer and System Sciences), University of Genova. Since 1996, he is Assistant Professor at DSEA (Department of Electrical System and Automation), University of Pisa, Italy. His research interests include model identification, parameter estimation, inverse problems, model-based signal processing and control, and their application. He participates in the IEEE Control Systems and Ocean Engineering Societies. In 1991, Prof. Caiti received the Cameli Award of the Italian Association of Electrical Engineers (AEI)
Sérgio M. Jesus received the "Doctorat Es-Sciences" in engineering sciences from the University of Nice, France, in 1986.

From 1985 to 1992 , he was a staff scientist at the SACLANT Undersea Research Centre, La Spezia, Italy in the Ambient Noise and Signal Processing Groups. During that period, he was involved with underwater acoustic field noise directionality and early studies on target detection using matched field processing. In 1992, he joined the Electronics and Computation Department at the University of Algarve, Faro, Portugal, where he is currently Associate Professor. His interests are underwater acoustics signal processing, modelbased inversion, ocean and seafloor tomography and their applications. He is member of the IEEE Signal Processing Society, EURASIP, and of the Acoustical Society of America.

Age Kristensen received the M.Sc. and Ph.D. degrees from the Institute of Technology in Trondheim, Norway, in 1976 and 1983, respectively.

During this time, he was employed in the Underwater Acoustics group, Electronics Research Laboratories (ELAB), in Trondheim, where he worked in sonar technology, hydroacoustic transducers, digital and analog electronics design and applications of acoustic methods in fishery research. Since 1983 he has worked for the Petroleum Technology Research Institute, Trondheim, (1983-84), Southwest Research Institute, San Antonio, TX, (1984), IKU, Trondheim, (1985-86), Statoil R\&D Centre, Trondheim (1986-90), all involving topics in borehole seismics, well logging instrumentation, and mathematical modeling of acoustics and electromagnetics wave propagation in boreholes. He was assigned to the SACLANT Undersea Research Centre, La Spezia, Italy, from 1990-95, and was Head of the Seafloor Acoustics Group, involved in geophysical mapping of the upper sediments. He is currently Department Manager for Geophysics at Statoil R\&D Centre, Trondheim. Dr. Kristensen is a member of the Acoustical Society of America. 